\title{
Shaping the Digital Twin for Design and Production Engineering
}

\author{
Benjamin Schleich ${ }^{\mathrm{a}}$, Nabil Anwer ${ }^{\mathrm{b}}$, Luc Mathieu $^{\mathrm{b}}$, Sandro Wartzack ${ }^{\mathrm{a}}$ \\ ${ }^{a}$ Chair of Engineering Design, Friedrich-Alexander-Universität Erlangen-Nürnberg, \\ Martensstrasse 9, 91058 Erlangen, Germany. \\ ${ }^{b}$ LURPA, ENS Cachan, Univ. Paris-Sud, Université Paris-Saclay, \\ 94235 Cachan, France.
}

\begin{abstract}
:
The digitalization of manufacturing fuels the application of sophisticated virtual product models, which are referred to as digital twins, throughout all stages of product realization. Particularly, more realistic virtual models of manufactured products are essential to bridge the gap between design and manufacturing and to mirror the real and virtual worlds. In this paper, we propose a comprehensive reference model based on the concept of Skin Model Shapes, which serves as a digital twin of the physical product in design and manufacturing. In this regard, model conceptualization, representation, and implementation as well as applications along the product life-cycle are addressed.
\end{abstract}

\section{Keywords:}

Design, Tolerancing, Digital Twin.

\section{Introduction}

In today's highly competitive markets, the ambitions for shortening the time to market and for increasing the product development performance fuel the application of sophisticated virtual product models, which are frequently referred to as digital twins. Enabled by the digitalization of manufacturing, cyber-physical production systems, model-based system engineering, and a growing endeavour for data gathering and processing, these models are increasingly enriched with production and operation data. Moreover, they allow the efficient prediction of the effects of product and process development as well as operating and servicing decisions on the product behaviour without the need for costly and time-expensive physical mockups [1-3]. Particularly in design, such realistic product models are essential to allow the early and efficient assessment of the consequences of design decisions on the quality and function of mechanical products. However, current approaches to the implementation of digital twins lack of a conceptual basis, which hinders the applicability of the digital twin vision to various activities in design and production engineering. Motivated by this need, this paper proposes a comprehensive reference model, which serves as a digital twin of the physical product in design and production engineering. In this regard, important model properties, such as scalability, interoperability, expansibility, and fidelity, as well as different operations on this reference model along the product life-cycle, such as composition, decomposition, conversion, and evaluation are addressed. Moreover, the application of this reference model to geometrical variations management is highlighted.

The paper is structured as follows. In the next section, the vision of the digital twin and its evolution is reviewed. After that, a comprehensive reference model for the digital twin is introduced, which is then applied to geometrical variations management. Finally, a conclusion and an outlook are given. 


\section{The Vision of the Digital Twin}

It has been argued, that "the vision of the digital twin itself refers to a comprehensive physical and functional description of a component, product or system, which includes more or less all information which could be useful in all - the current and subsequent - lifecycle phases" [4]. In the following, the evolution of this vision is briefly illustrated, recent definitions of the digital twin are discussed, and several viewpoints on it are addressed.

\subsection{The Origin and Evolution of the Digital Twin}

Prior to the industrial revolution, physical artefacts were predominantly manufactured by artisans resulting in unique instances of a given template. However, with the introduction of the concept of interchangeable parts in the $18^{\text {th }}$ century, the way products were designed and manufactured rapidly changed as companies began to strive for building copies of their products in mass production. Recently, the paradigm of mass customization aims at combining these two established manufacturing concepts to achieve low unit costs for customized products.

However, though such manufacturing paradigms allow the fabrication of large quantities of similar, i. e. customized, parts or products, these manufactured instances are mere unrelated copies. In contrast to that, the idea of building a twin refers to producing a copy of a part or product and using it for reasoning about other instances of the same part or product - thus establishing a relation between multiple copies. This idea is said to originate from NASA's Apollo program, "where at least two identical space vehicles were built to allow mirroring the conditions of the space vehicle during the mission" [5].

However, over the past decades, advancements in computer technology enabled establishing increasingly sophisticated virtual models of physical artefacts as well as the fusion of such models for systems engineering [6]. These models not only serve for the design verification and validation [7], but are also increasingly used as the master product model comprising the model-based definition of required product characteristics [8]. Moreover, the evolvements in "microchip, sensor and IT technologies" [9] paved the way for the advent of smart products, which track and communicate their operating conditions and thus allow to "feed" their product models with data about their status, such as environmental conditions and loads. $s$ Beside this, modern sensing routines, which go even beyond geometrical measurement and scanning, allow the easy, quick, and reliable collection of large sets of data from physical artefacts. Pattern recognition, data mining, deep learning, reverse engineering, and other data analysis approaches make use of these data sets and unveil dependencies between product, process, and operational characteristics that used to be hidden. The vast developments in simulation technology as well as the increasing possibilities for gathering and exchanging data from products thus allowed for building virtual twins of physical products, which finally led to the present understanding of the "digital twin" vision. Probably the first definition of it was given by NASA in their integrated technology roadmap (Technology Area 11: Modeling, Simulation, Information Technology \& Processing Roadmap; 2010), which has been slightly adapted in [10]: "A Digital Twin is an integrated multiphysics, multiscale, probabilistic simulation of an as-built vehicle or system that uses the best available physical models, sensor updates, fleet history, etc., to mirror the life of its corresponding flying twin" (Fig. 1). With some similarity to this, Grieves defines the digital twin as "a set of virtual information constructs that fully describes a potential or actual physical manufactured product from the micro atomic level to the macro geometrical level" [11]. 


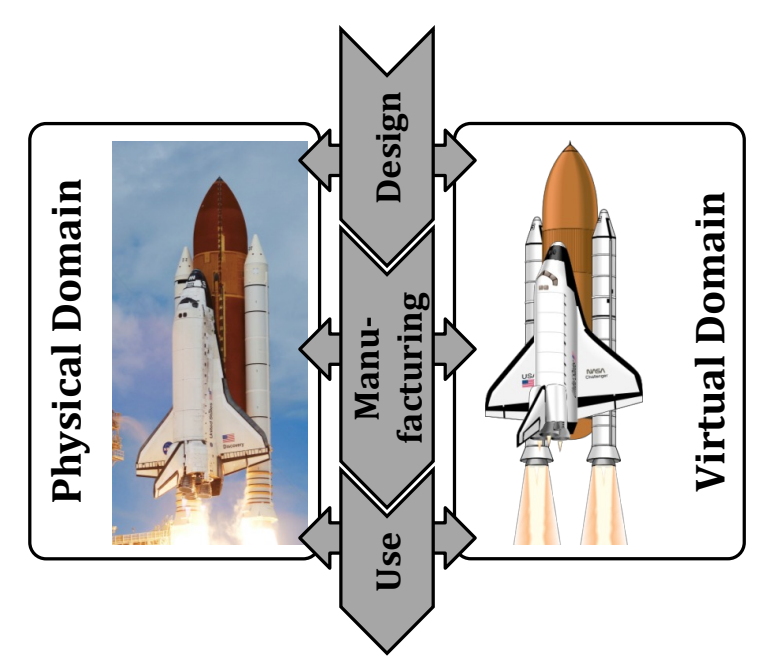

Fig. 1. The Vision of the Digital Twin throughout the Product Life-Cycle following [11].

\subsection{Viewpoints on the Digital Twin}

Based on these definitions, it can be found, that there exist different viewpoints on the vision of the digital twin. In this context, it has been argued, that "from a simulation point of view the digital twin approach is the next wave in modelling, simulation and optimization technology" [5]. However, the digital twin is not one complete model of the physical product, but a set of linked operation data artefacts and simulation models, which are of suitable granularity for their intended purpose and evolve throughout the product lifecycle [4]. Thus, the digital twin not only serves representation purposes but is also applicable for making predictions about the expected product behaviour [4], while the granularity of the simulation models fits to their purpose and evolves from early design stages, where simple product models are used to decide about product concepts, to detail design, where sophisticated simulation models support the dimensioning and design of parts and subassemblies.

As a result, from a product life-cycle management point of view, there is a strong need to integrate all lifecycle data artefacts into a comprehensive management system, which can be used by various actors for querying data, such as through-life performance information, and making predictions about the physical product from the digital twin for design optimization and manufacturing system improvement [5].

\subsection{Current Industrial Understanding and Applications}

Different understandings of the "digital twin" can be observed in industrial practice, particularly by PLM software vendors. In this regard, it has been argued, that PTC is focusing on establishing a link between the virtual product model and the physical part to increase the manufacturing flexibility and competitiveness, while Dassault Systèmes targets the product design performance by creating a platform that enables designers to virtually build and interact with complex systems. Moreover, the digital twin understanding of General Electric focuses on forecasting the health and performance of their products over lifetime, whereas SIEMENS strives for improved efficiency and quality in manufacturing by exploiting the possibilities of today's manufacturing digitalization in the context of Industry 4.0. TESLA aims at developing a digital twin for every built car, hence enabling synchronous data transmission between the car and the factory, while other companies increasingly use complex product models to boost the immersion in virtual and augmented reality applications. 


\subsection{Synthesis and Discussion}

As it has been highlighted, the vision of the digital twin evolved during the last decades and describes the vision of a bi-directional relation between a physical artefact and the set of its virtual models. In this context, the virtual "twinning", i.e. the establishment of such relations between physical parts and their virtual models, enables the efficient execution of product design, manufacturing, servicing, and various other activities throughout the product life-cycle. Particularly in design, such digital twins allow the check for conformance of the product specifications with the design intent and customer requirements.

However, as it has been reported in scientific literature and in practice, current limitations to the implementation of the vision of the digital twin are insufficient possibilities for synchronization between the physical and the digital world to establish closed loops, the missing of high-fidelity models for simulation and virtual testing at multiple scales, the lacking uncertainty quantification for such models, the difficulties in the prediction of complex systems, as well as the challenges for gathering and processing large data sets. Indeed, these challenges can only be mastered based on a sound conceptual framework and a comprehensive reference model for the digital twin.

\section{A Reference Model for the Digital Twin}

The need for realistic virtual product models for design and manufacturing activities is ubiquitous. However, current approaches to the implementation of digital twins lack of a conceptual basis. This hinders the applicability of the implementations to various activities in design and production engineering and hence handicaps the efforts for building integrated digital twins of physical artefacts. Motivated by this shortcoming, we propose a reference model for the digital twin, which can be considered as a template for the implementation of digital twins for specific applications while ensuring important model properties, such as model scalability, interoperability, expansibility, and fidelity.

\subsection{Background}

The conceptual background of the reference model is borrowed from classical solid modelling and recent developments in ISO standards for Geometrical Product Specifications (GPS), which offer a widely acknowledged framework for the modelling, specification, and verification of product geometry. Solid modelling has introduced the abstraction-representation schema to conceptualize the idealized physical product, and to implement it on computers with a suitable representation. ISO GPS standards evolved from industrial best-practices and examples to more rigorous concepts and a language with consistent syntax and semantics with the aim of transferring them into the age of digitalization. These conceptualization efforts have led to the development of the GeoSpelling "language" [12] which is based on sound concepts to allow the unambiguous definition of geometrical specifications and to mirror specification and verification operations on the grounds of a duality principle. One of these basic concepts is the Skin Model, which is an abstract model of the physical interface between a workpiece and its environment [13]. The Skin model permits a mental model and thought experiments to develop a theoretical inspection procedure, hence connecting the specification world to the physical world. Furthermore, several mirrored operations and uncertainties (ambiguities, method uncertainty, implementation uncertainty) were defined [14]. 


\subsection{Model Abstraction and Representation}

The idea behind the comprehensive reference model is to extend and transfer the above conceptual framework and to convey the scientific fundamentals of the geometrical product specification language to the vision of the digital twin. The novelty of this reference model is the particular focus on the "twinning" between the physical and the virtual realm. In this regard, we propose to endow the digital twin with an abstract model that comprises all characteristics and fully describes the physical twin at a conceptual level throughout the whole product life-cycle. Based on this abstract model thoughts experiments can be performed, which allows to capture and understand as well as to clearly describe the physical twin, its behaviour and its environment at an abstract level.

The experiences from GPS standardization and recent research efforts on tolerancing led to the insight that only the differentiation between the real workpiece, its abstract and conceptual model (Skin Model), and the virtual representation of this abstract model allows for a clear understanding as well as a forward-looking language for geometrical variations management. Consequently, the concept of Skin Model Shapes has been developed for the virtual representation of the Skin Model based on discrete geometry representation schemes [15]. With similarity to this, we propose a differentiation between the abstraction of the digital twin and its representation (Fig. 2). In this context, the model representation may depend on the particular application and can involve various representation and modelling paradigms.

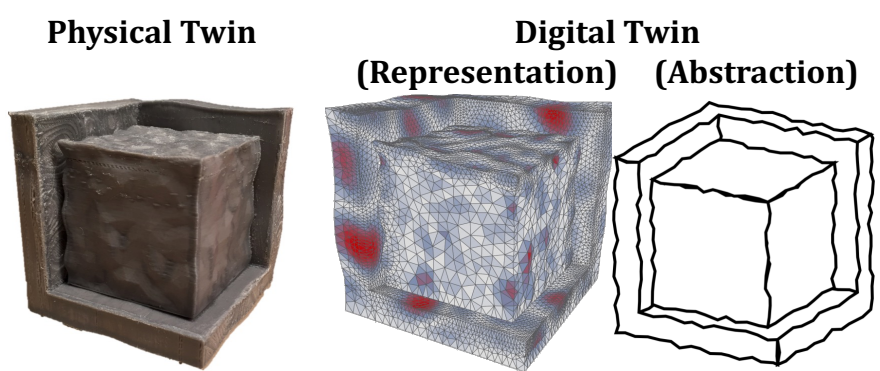

Fig. 2. Physical Twin and Reference Model for the Digital Twin (Representation and Abstraction).

The differentiation between a conceptual model and its virtual representation for the digital twin allows achieving important model properties (Table 1), such as model scalability, interoperability, expansibility, and fidelity. In this regard, the abstract model description enables the definition of model operations at different scales, while models for different interfaced parts and subassemblies of a product can be conceptually queried and digital product models can be expanded to system models. 
Table 1 Properties of the Reference Model for the Digital Twin.

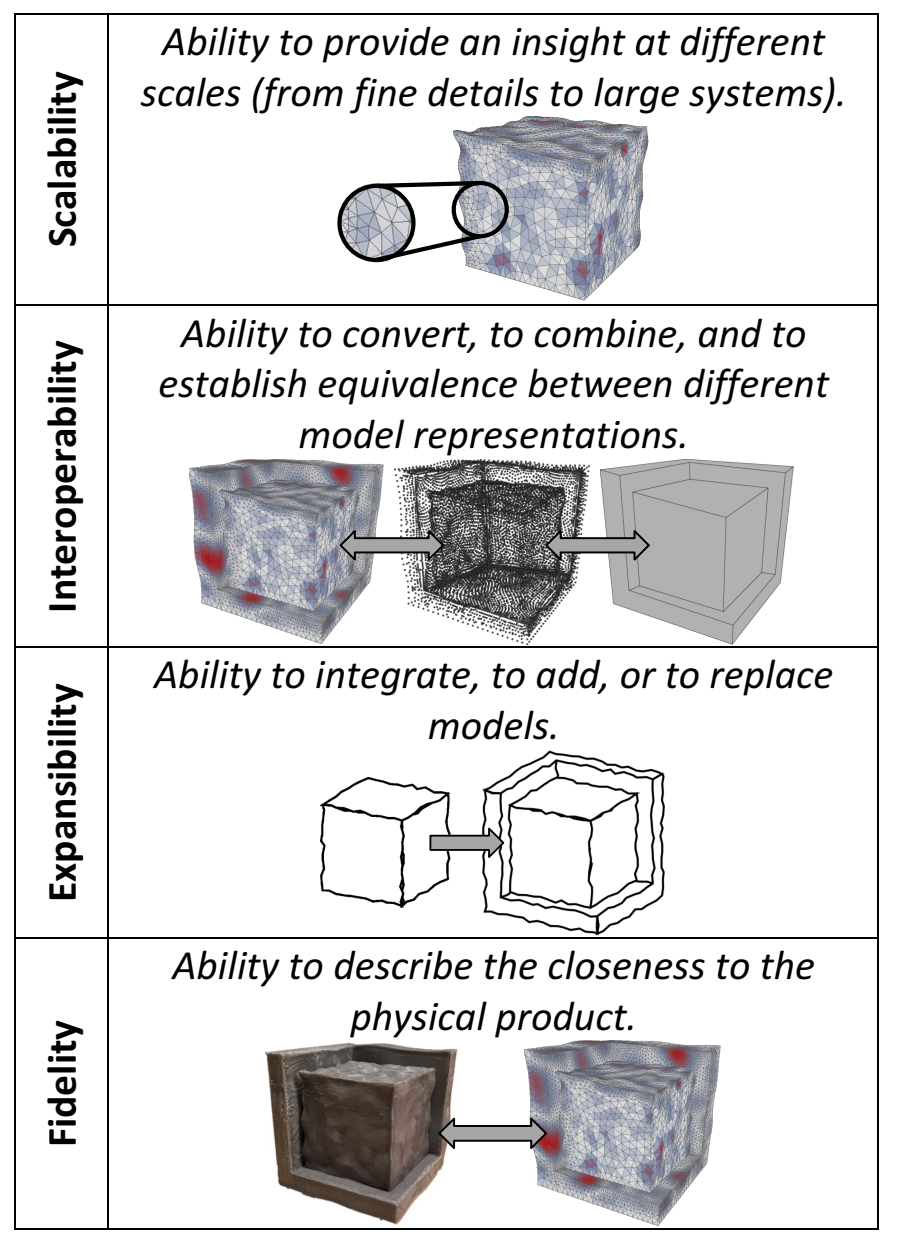

\subsection{Operations on the Reference Model}

Beside the Skin Model, the duality principle is anchored in the ISO GPS standards. It allows the differentiation between a specification operator, which covers a set of operations that are used to define certain geometrical specifications on the Skin Model, and the verification operator, which mirrors the specification operator and comprises the operations that are required to verify the specifications on the real part. Though the verification operator mirrors the specification operator, there will always remain uncertainties whether the verification operations fully comply with the specification operator.

This duality principle as well as the concept of uncertainties is also conveyed to the reference model for the digital twin. In this regard, the application of a digital twin to different scenarios and tasks in design or production engineering dictates to apply certain operations on the digital twin. These operations depend on the specific application and are backed by physical counterparts (such e.g. manufacturing or assembly operations). The abstraction of the digital twin allows describing these operations on an abstract level, while their virtual "representation" is performed employing specific simulation models. Due to the nature of simulation models, obviously, uncertainties will remain between the abstract description of a certain operation and its representation. We propose here to classify the set of operations in four broad categories, namely conversion, composition, decomposition, and evaluation.

Moreover, the "twinning" between the physical world and the virtual world can be considered as observation and prediction phases at a conceptual level (Fig. 3). While the information transfer from the physical to the digital twin is related to the observation and sensing of the physical twin, the information transfer from the digital to the physical twin originates from scientific assumptions, simulation and virtual 
testing models, with proper handling of uncertainty, used to predict certain characteristics and the behaviour of the physical twin.

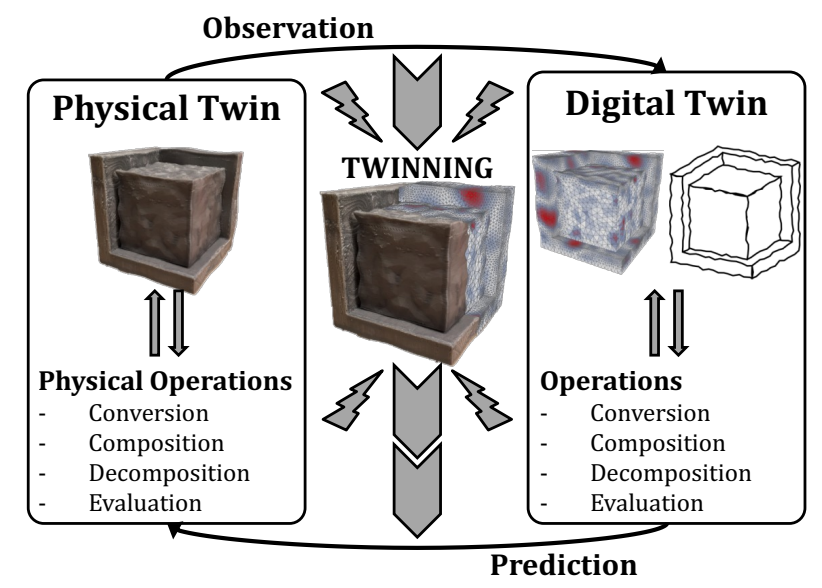

Fig. 3. Different Operations on the Physical and the Digital Twin throughout the Product Life-Cycle.

\section{A Digital Twin for Geometrical Variations Management}

The aim of geometrical variations management is to ensure that the required geometrical characteristics of the product are met despite the presence of geometrical part deviations. The implementation of a digital twin for geometrical variations management hence requires the consideration of all different views throughout the product life-cycle that relate to the specification, generation, verification, and propagation of geometrical deviations. For each of these views and applications, specific operations on the digital twin are defined and mirrored by corresponding virtual representations. For this purpose, the set of operations defined in GPS [16] are exploited and mapped to those that operate on the reference model.

The concept of Skin Model Shapes as a paradigm shift in computer-aided tolerancing and geometrical variations management [17] serves as a first approach to connect these views and operations in a comprehensive model that incorporates manufacturing process planning and inspection process planning (Fig. 4). Thus, an important step towards the "twinning" between the physical world and a virtual model for a digital twin in geometrical variations management has been taken, which serves as a sound basis for ongoing research.

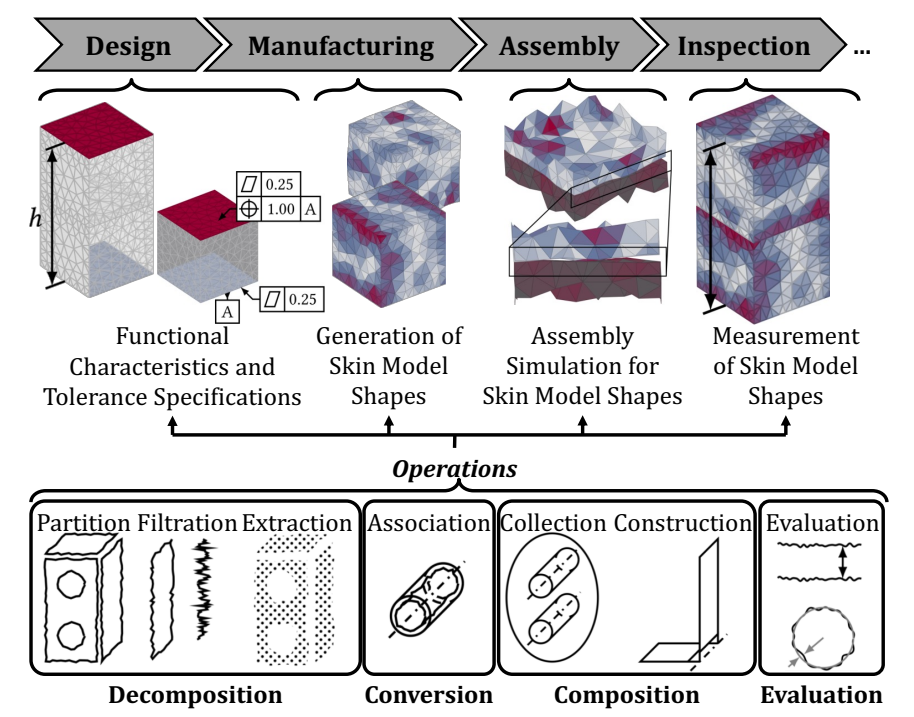

Fig. 4. Digital Twin for Geometrical Variations Management 


\section{Conclusion and Outlook}

The ability for assessing the consequences of product, process, and servicing decisions employing virtual models is an important competitive factor for modern manufacturing companies. The vision of the digital twin refers to an entangled relation between a physical artefact and the set of such virtual models.

In this paper, the history and evolvement of the vision of the digital twin as well as its current understanding and applications have been portrayed. Beside this, current limitations to the implementation of digital twins have been discussed and a comprehensive reference model for the digital twin in design and production engineering has been highlighted. Particularly, this reference model enables the clear differentiation between a conceptual model and its virtual representation for the digital twin. The application of this reference model to geometrical variations management has also been sketched. The benefit of this paper for research as well as industrial applications is the provision of a first theoretical and conceptual framework for the digital twin, which is to be enriched by the design and production engineering community in the future and which may lead to a scientific discussion about the vision of the digital twin and its implementation throughout the product life-cycle.

\section{References}

[1] Leu MC, EIMaraghy H, Nee A, Ong SK, Lanzetta M, Putz M, Zhu W, Bernard A (2013) CAD model based virtual assembly simulation, planning and training. CIRP Ann. 62(2):799-822.

[2] Roy R, Stark R, Tracht K, Takata S, Mori M (2016) Continuous maintenance and the future Foundations and technological challenges. CIRP Ann. 65(2):667-688.

[3] Altintas Y, Kersting P, Biermann D, Budak E, Denkena B, Lazoglu I (2014) Virtual process systems for part machining operations. CIRP Ann. 63(2):585-605.

[4] Boschert S, Rosen R (2016) Digital Twin - The Simulation Aspect. In Hehenberger P, Bradley D (eds.): Mechatronic Futures: Challenges and Solutions for Mechatronic Systems and their Designers, Springer International Publishing, 2016, 59-74.

[5] Rosen R, von Wichert G, Lo G, Bettenhausen KD (2015) About The Importance of Autonomy and Digital Twins for the Future of Manufacturing, IFAC-PapersOnLine 48(3): 567-572.

[6] Lu SC-Y, Li D, Cheng J, Wu CL (1997) A Model Fusion Approach to Support Negotiations during Complex Engineering System Design. CIRP Ann. 46(1):89-92.

[7] Maropoulos P, Ceglarek D (2010) Design verification and validation in product lifecycle. CIRP Ann. 59(2):740-759.

[8] Quintana V, Rivest L, Pellerin R, Venne F, Kheddouci F (2010) Will Model-based Definition replace engineering drawings throughout the product lifecycle? A global perspective from aerospace industry. Comput. Ind. 61(5):497-508.

[9] Abramovici M, Göbel JC, Dang HB (2016) Semantic data management for the development and continuous reconfiguration of smart products and systems. CIRP Ann. 65(1):185-188.

[10] Glaessgen EH, Stargel DS (2012) The Digital Twin Paradigm for Future NASA and U.S. Air Force Vehicles. Proceedings of the 53rd AIAA Structures, Structural Dynamics and Materials Conference, 2012, Paper no. 1818.

[11] Grieves M, Vickers J (2017) Digital Twin: Mitigating Unpredictable, Undesirable Emergent Behavior in Complex Systems. In Kahlen F-J, Flumerfelt S, Alves A (eds.): Transdisciplinary Perspectives on Complex Systems: New Findings and Approaches, Springer International Publishing, 2017, 85-113.

[12] Mathieu L, Ballu A (2003) GEOSPELLING: a common language for Geometrical Product Specification and Verification to express method uncertainty. In Wilhelm R (ed): Proc. of the 8th CIRP Int. Seminar on Computer Aided Tolerancing. 70-79. 
[13] Anwer N, Ballu A, Mathieu L (2013) The skin model, a comprehensive geometric model for engineering design. CIRP Ann. 62(1):143-146.

[14] Nielsen HS (2013) Recent developments in International Organization for Standardization geometrical product specification standards and strategic plans for future work. Proceedings of the Institution of Mechanical Engineers, Part B: Journal of Engineering Manufacture 227(5):643-649.

[15] Schleich B, Anwer N, Mathieu L, Wartzack S (2014) Skin Model Shapes: A new paradigm shift for geometric variations modelling in mechanical engineering. Comput.-Aided Des. 50:1-15.

[16] ISO 17450-1:2011, Geometrical Product Specifications (GPS) - General concepts - Part 1: Model for geometrical specification and verification.

[17] Schleich B, Anwer N, Mathieu L, Wartzack S (2016) Status and Prospects of Skin Model Shapes for Geometric Variations Management. Procedia CIRP 43:154-159. 